\title{
Panorama das Publicações Nacionais sobre Autismo, Educação e Tecnologia
}

\author{
Williby Ferreira $^{1}$, Rafael Cordeiro', Yuska P. C. Aguiar ${ }^{2,3}$, Juliana Saraiva ${ }^{2}$, \\ Carole Tardif ${ }^{3}$, Edith Galy ${ }^{4}$ \\ ${ }^{1}$ Centro de Informática, UFPB - Campus I, João Pessoa, PB, Brasil \\ ${ }^{2}$ Departamento de Ciências Exatas, UFPB - Campus IV, Rio Tinto, PB, Brasil \\ ${ }^{3}$ Aix-Marseille Université, Centre PsyClé, Aix-en-Provence, PACA, França \\ ${ }^{4}$ l'Université Nice Sophia-Antipolis, LAPCOS, PACA, França \\ willibyferreiralppgi.ci.ufpb.br, cordeirof.rafaelegmail.com, \\ yuska@dcx.ufpb.br, julianajag@gmail.com, carole.tardif@univ-amu.fr, \\ edith.galyeunice.fr
}

\begin{abstract}
The symptoms' heterogeneity of people with ASD requires that therapeutic and educational interventions be case-specific. In Brazil, the government's guidelines aim the educational inclusion of this population in regular school with technology support. In this scenario, it is relevant a national scientific production mapping that have been addressing "Autism, Education and Technology" for compiling information aiming a research collaboration network. Therefore, a literature review was conducted, contemplating 33 articles. A prevalence of educational software solutions for literacy of children with ASD was identified, and these studies were published more frequently in Alagoas and Rio Grande do Sul.
\end{abstract}

Resumo. A heterogeneidade dos sintomas das pessoas com TEA exige que as intervenções terapêuticas e educacionais sejam específicas para cada caso. No Brasil, as diretrizes do governo visam a inclusão educacional dessa parte da população em escola regular com o auxílio de tecnologia. Neste cenário, é relevante mapear a produção científica nacional sobre "Autismo, Educação e Tecnologia" para compilar informações sobre as pesquisas realizadas e favorecer a criação de uma rede colaborativa. Logo, a partir de uma revisão de literatura, contemplando 33 artigos, identificou-se a predominância de soluções de softwares educativos para alfabetização de crianças com TEA, sendo os estudos mais frequentes em Alagoas e no Rio Grande do Sul.

\section{Introdução}

O Transtorno do Espectro Autista $\left(\right.$ TEA $\left.^{1}\right)$ se caracteriza pela persistência no déficit de comunicação e interação social e pelos padrões de comportamento, de interesse e de atividades que são restritos e repetitivos [APA, 2013]. O termo "Espectro" representa a pluralidade de sintomas e comprometimentos possíveis e engloba o Transtorno Autista, a Síndrome de Asperger e o Transtorno Invasivo do Desenvolvimento Não-Especificado. Os múltiplos sintomas de TEA e as comorbidades comumente associadas (deficiência

${ }^{1}$ No texto, os termos TEA e Autismo serão considerados sinônimos e ambos contemplam o Transtorno Autista, a Síndrome de Asperger e o Transtorno Invasivo do Desenvolvimento Não-Especificado. 
VII Congresso Brasileiro de Informática na Educação (CBIE 2018)

Anais do XXIX Simpósio Brasileiro de Informática na Educação (SBIE 2018)

intelectual, dificuldade de aprendizado, etc.) colocam em evidência a realidade da heterogeneidade inter e intra-indivíduo. Logo, atividades do cotidiano e as intervenções terapêuticas e educacionais devem ser adaptadas para cada caso, favorecendo o desenvolvimento do indivíduo e ampliando suas capacidades [de Matos Cano, 2016].

No Brasil, a Lei no 12.764 [Brasil, 2012] institui a Política Nacional de Proteção dos Direitos da Pessoa com TEA e visa garantir para esta parte da população os mesmos direitos legais das pessoas com deficiência. $\mathrm{Na}$ educação, alunos com TEA devem estar incluídos na rede regular de ensino e recebendo "Atendimento Educacional Especializado", sendo este responsável pela identificação, elaboração e organização de recursos pedagógicos e de acessibilidade [Nunes et al., 2013]. De acordo com as diretrizes do Governo Federal [Ministério da Saúde, 2013] é esperado que tais recursos considerem as práticas educativas e métodos de comunicação alternativa que são amplamente adotados, a exemplo de: ABA (Applied Behavior Analysis), TEACCH (Treatment and Education of Autistic and related Communication handicapped Children), CAA (Comunicação Alternativa e Aumentada) e PECS (Picture Exchange Communication System). Em consonância com [Rogé, 2010], o mesmo documento evidencia a importância do uso de tecnologia para potencializar inclusão escolar.

A realidade nacional estima 1,5 milhões de brasileiros com Transtorno de Espectro Autista [Mello et al., 2013] com crescimento no número de diagnósticos efetivados [Rios et al., 2015]. No contexto internacional o binômio "Autismo e Tecnologia" [Grynszpan et al., 2014; Virnes et al., 2015], assim como "Autismo, Educação e Tecnologia" [Fletcher-Watson, 2014] têm sido discutidos. Este estudo tem por objetivo identificar como as pesquisas desenvolvidas e disseminadas em eventos acadêmicos nacionais têm contemplado a tríade "Autismo, Educação e Tecnologia". Para tanto, realizou-se uma revisão de literatura considerando publicações nacionais em anais de conferências e em revistas que abordam temas relacionados à Inclusão, Acessibilidade, Educação e Tecnologia.

\section{Metodologia}

O protocolo adotado na revisão de literatura contempla (i) um conjunto de questões de pesquisa; (ii) as bases dados contempladas (repositórios de artigos); as palavras chaves da pesquisa (strings de busca); (iii) um conjunto de critérios de inclusão e exclusão; (iv) o método de extração de dados e, (v) análise de dados.

O escopo da pesquisa versa sobre as publicações nacionais transversais à tríade "Autismo, Educação e Tecnologia" para responder às seguintes questões de pesquisa: QP01: Quais são as soluções tecnológicas descritas? QP02: Quem são os potenciais usuários destas soluções? QP03: Quais competências dos usuários são alvo das soluções? QP04: Quais são os contextos de uso das soluções? QP05: Quais são as bases teóricas que apoiam o desenvolvimento das soluções? e QP06: Qual é a distribuição geográfica dos artigos?

Diante da multidisciplinaridade envolvida no estudo, foram considerados repositórios de publicações nacionais que indexam artigos nas áreas de Inclusão, Acessibilidade, Educação e Informática, a saber: (i) na Biblioteca Digital Brasileira de Computação (BDComp); (ii) Portal de Publicações da Comissão Especial de Informática na Educação ( $\underline{\mathrm{CEIE}})$; e (iii) no Portal da Comissão Especial de Interação HumanoComputador ( $\underline{\mathrm{IHC}})$. As revistas $\underline{\mathrm{RENOTE}}$ e $\underline{\mathrm{RITA}}$ também foram consultadas. A seleção 
VII Congresso Brasileiro de Informática na Educação (CBIE 2018)

Anais do XXIX Simpósio Brasileiro de Informática na Educação (SBIE 2018)

inicial dos artigos se deu via busca manual nos títulos das publicações pelas palavraschaves: "Autismo", "Autista", "TEA" ou "ASD” (Autism Spectrum Disorder).

$\mathrm{Na}$ primeira etapa de filtragem dos artigos, a partir do acesso e da leitura do resumo dos mesmos, foram aplicados os critérios de exclusão para os artigos: duplicados, estudos secundários (revisões e mapeamento da literatura) ou indisponíveis (pagos ou não encontrados). Na etapa seguinte, com a leitura completa dos artigos, foram aplicados os critérios de inclusão, ou seja, artigos: que descrevem soluções tecnológicas (ou de desenvolvimento de tecnologia) para pessoas com TEA, escritos em português ou inglês e com conteúdo suficiente para os objetivos da revisão. Em ambas as etapas participaram dois pesquisadores e os conflitos foram discutidos com um terceiro pesquisador.

As informações de interesse extraídas dos artigos foram: (i) referência completa, (ii) conferências ou revista de origem; (iii) ano de publicação; (iv) nomes e (v) filiação dos autores. Além disso, foram transcritas as respostas dos artigos às questões de pesquisa. Cada artigo foi associado a um ID (A01: Artigo 01), em ordem crescente de acordo com o ano de publicação. A compilação e análise são descritas na seção seguinte. As informações completas estão disponíveis no link: goo.gl/HFCXbJ.

\section{Resultados e Discussão}

O processo de busca manual resultou em um conjunto inicial de 52 artigos, sendo mais numerosas as publicações indexadas na base de dado CEIEE (30/52), com maior frequência nos meios de publicação SBIE (15), CBIE (08) e IHC (08). Após a aplicação dos critérios de exclusão e inclusão, o total de 33 artigos foi mantido para análise (aproveitamento de 64.7\%). Os eventos SBIE, CBIE e IHC são os mais frequentes após a filtragem, evidenciando a pertinência do tema nestes. O quantitativo dos artigos por origem e etapa da revisão de literatura está disponível na Figura 1.

\begin{tabular}{|c|c|c|c|c|}
\hline \multicolumn{2}{|c|}{ Origem do Artigo } & \multicolumn{3}{|c|}{ Número de Artigos } \\
\hline Base de dados & Meio & Retorno Inicial & Etapa 1 & Etapa 2 \\
\hline \multirow{4}{*}{ CEIEE } & SBIE & 15 & 11 & 11 \\
\hline & CBIE & 08 & 06 & 06 \\
\hline & WIE & 04 & 04 & 03 \\
\hline & RBIE & 03 & 03 & 01 \\
\hline \multirow{6}{*}{ BDBComp } & WTIC & 02 & 02 & 02 \\
\hline & SBSI & 02 & 02 & 01 \\
\hline & SBSC & 01 & 01 & 01 \\
\hline & $\mathrm{Cl}$ & 01 & 01 & 01 \\
\hline & WIM & 01 & 00 & 00 \\
\hline & PLN & 01 & 00 & 00 \\
\hline CEIHC & IHC & 08 & 05 & 04 \\
\hline \multicolumn{2}{|l|}{ RENOTE } & 05 & 03 & 02 \\
\hline \multirow{2}{*}{\multicolumn{2}{|c|}{ RITA }} & 01 & 01 & 01 \\
\hline & & 52 & 39 & 33 \\
\hline
\end{tabular}

Figura 1. Quantitativo de artigos durante a execução do protocolo de pesquisa²

\footnotetext{
${ }^{2}$ SBIE (Simpósio Brasileiro de Informática na Educação); CBIE (Congresso Brasileiro de Informática na Educação); WIE (Workshop de Informática na Educação); RBIE (Revista Brasileira de Informática na Educação); WTIC (Workshop de Tecnologia da Informação e Comunicação); SBSI (Simpósio Brasileiro de Sistemas de Informação); SBSC (Simpósio Brasileiro de Sistemas Colaborativos); CI (Cadernos de Informática); WIM (Workshop de Informática Médica); PLN (Processamento de Linguagem Natural)
} 
VII Congresso Brasileiro de Informática na Educação (CBIE 2018)

Anais do XXIX Simpósio Brasileiro de Informática na Educação (SBIE 2018)

As publicações consideradas na pesquisa compreendem o período de 2002 a 2017, havendo uma concentração de 22 destas nos três últimos anos e sendo remarcável a ausência de artigos para seis dos dezesseis anos considerados (Figura 2).

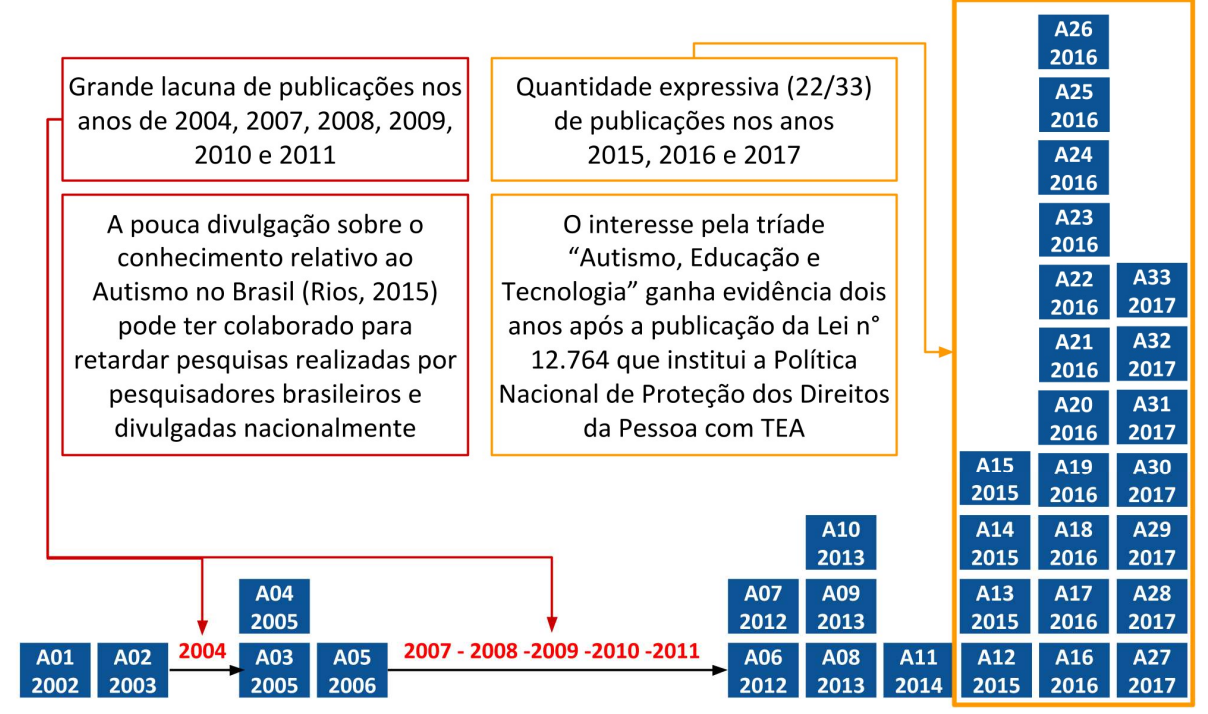

Figura 2. Distribuição dos artigos por ano de publicação

\subsection{Soluções para Pessoas com TEA (QP01)}

De forma a especificar quais foram as soluções descritas nos artigos, a Figura 3 contém o conjunto dos identificadores dos artigos com os respectivos nomes das soluções descritas. As duplas de artigos [A07 A16] e [A14 A19], embora tenham abordado a mesma solução, o fizeram sob aspectos distintos, tornando as informações complementares e relevantes para o estudo.

\begin{tabular}{|l|l|l|l|l|l|}
\hline A01 & SACA & A12 & ABC Autismo & A23 & Fotos Selfs em Tablets \\
\hline A02 & HagáQuê & A13 & Blog & A24 & HangAut Game \\
\hline A03 & Descobrindo Emoções & A14 & TEO & A25 & ProAut \\
\hline A04 & MsChat da Microsoft & A15 & Não nomeada & A26 & Não nomeada \\
\hline A05 & Ambiente digital de aprendizagem & A16 & SCALA $^{\text {a }}$ & A27 & mTEA \\
\hline A06 & WorldTour & A17 & GAIA $^{c}$ & A28 & LIA \\
\hline A07 & SCALA & A18 & Vitula Assistiva & A29 & Protótipo (não nomeado) \\
\hline A08 & PAR (Peço, Ajudo, Recebo) & A19 & TEO & A30 & Várias soluções \\
\hline A09 & ComFim & A20 & Não nomeada & A31 & Protótipo (não nomeado) \\
\hline A10 & Protótipo (não nomeado) & A21 & Não nomeada & A32 & SwAspie \\
\hline A11 & Design Probes & A22 & Litera Azul & A33 & Protótipo (não nomeado) \\
\hline
\end{tabular}

Legenda: 'Sistema de Comunicação Alternativo para o Letramento de pessoas com Autismo; 'bratar, Estimular e Orientar; 'Guia de Acessibilidade de Interfaces Web com foco em aspectos do Autismo; 'Letramento Interativo para Autistas; 'Color Monster, TP-Shapes, ABC Palavros, Doodiecast, Seasons, 123 Color, Aiello, Number Sequence.

Figura 3. Identificadores e nomes das soluções descritas nos artigos analisados

As soluções descritas nos artigos compreendem jogos (6/33), sites ou aplicações web (4/33), realidade aumentada (2/33), ambiente de aprendizagem (2/33) e diagnóstico de TEA (1/33). A maioria dos artigos consultados (17/33) não explicita o tipo de software da solução descrita. Para estes, adotamos o termo genérico "software". Três artigos (3/33) descrevem proposições metodológicas para auxiliar no desenvolvimento destas soluções 
VII Congresso Brasileiro de Informática na Educação (CBIE 2018)

Anais do XXIX Simpósio Brasileiro de Informática na Educação (SBIE 2018)

(Figura 4). Apenas quatro (4/33) artigos mencionam as plataformas a partir das quais as soluções tecnológicas são acessadas (mobile [A12 A19]; web e mobile [A16 A27]).

\section{Tipos de Soluções}

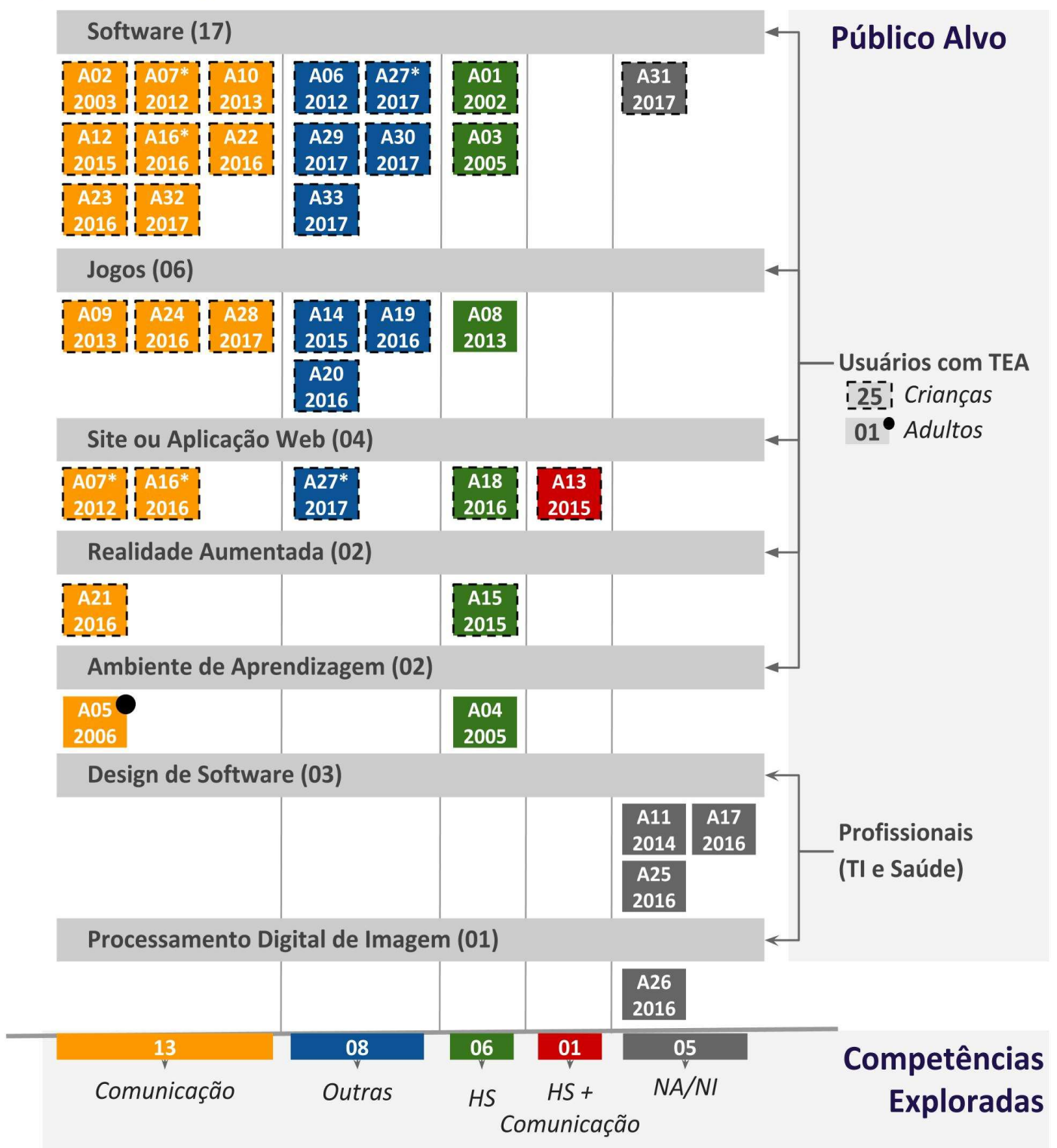

Legenda: NA: Não se Aplica; NI: Não Informado; HS: Habilidade Social; [A07, A16, A27] Duas soluções são propostas (software e aplicação web); Artigos sem borda correspondem aos trabalhos nos quais o público alvo não foi especificado

Figura 4. Artigos e o tipo de solução, o público alvo e as competências

\subsection{Público Alvo das Soluções (QP02)}

É possível identificar a expressividade de soluções destinadas ao público de pessoas com TEA (29/33), predominando o público infantil de usuários (25/33) (Figura 4). Este fato pode ser associado à importância de um diagnóstico prematuro (em torno dos três anos) seguido de intervenções terapêuticas e educacionais [Vieira et al., 2017]. Apesar da diferença entre a idade cronológica e a de desenvolvimento (real) das pessoas com TEA ser determinante para a escolha das práticas de intervenção a serem adotadas, os artigos não disponibilizam esta informação. Uma minoria de artigos (4/33) destina-se ao público de profissionais, contemplando desenvolvedores de software (3/33) cujos potenciais 
VII Congresso Brasileiro de Informática na Educação (CBIE 2018)

Anais do XXIX Simpósio Brasileiro de Informática na Educação (SBIE 2018)

usuários seriam pessoas com TEA e profissionais da área de saúde (1/33) que lidam com o diagnóstico de TEA. Colocando em evidência os artigos úteis para desenvolvedores de software destinados aos usuários com TEA, dois deles contemplam práticas de Design Participativo com o envolvimento de terapeutas [A11] e crianças com autismo [A25], e o terceiro [A17] propõe um conjunto de recomendações para acessibilidade Web considerando as particularidades das pessoas com Autismo.

\subsection{Competências Alvo das Soluções (QP03)}

As competências alvo das soluções tecnológicas destinadas aos usuários com TEA são: "Comunicação" (13/33), e "Habilidade Social" (6/33). Apenas um artigo contempla a junção das competências "Comunicação e Habilidade Social" (Figura 4). Considerando que os sintomas de TEA recaem sobre estas competências, é esperado que sejam contemplados nos estudos. Para a competência de "Comunicação" tem-se uma concentração de propostas direcionadas ao letramento e alfabetização [A10 A12 A21 A22 A24 A28 A32], seguida de estratégias de comunicação com base em imagens [A09 A23] e história em quadrinho [A02]. Para "Habilidade Social" destacam-se trabalhos sobre Interação social [A01 A04 A08 A15]. Além destes, outras diversas competências (8/33) foram contempladas: raciocínio, comunicação, percepção visual, concentração e de outras habilidades de forma geral [A14 A19], atividades lúdicas [A06], atividades educativas [A27 A33] (sendo o segundo para matemática), atividades diárias [A29] e exercícios de pareamentos [A30].

\subsection{Contexto de Uso (QP04) e Bases Teóricas das Soluções (QP05)}

O contexto de uso "Educacional" é o mais expressivo entre os artigos analisados neste estudo (Gráfico 1). Embora, hipoteticamente estes dados sejam reflexo das bases de busca utilizadas na pesquisa, é preciso ressaltar a importância de intervenções pedagógicas precoces (desde o diagnóstico) e contínuas para pessoa com TEA. O contexto de uso "Terapêutico" tem seis ocorrências. É relevante destacar que o limiar entre os dois contextos mencionados é tênue. Tanto o acompanhamento terapêutico tem sido inserido nas escolas para auxiliar a inclusão [Gomes e Machado, 2016], quanto é comum que intervenções terapêuticas adotem estratégias educativas. Diante da importância de estender as práticas educacionais e terapêuticas ao cotidiano da pessoa com Autismo, o contexto de uso "Geral” também é mencionado.

As bases teóricas descritas nos artigos possuem foco: (i) em métodos para propiciar a comunicação (CAA e PECS) ou para permitir a prática de intervenções educacionais e terapêuticas (ABA e TEACCH); ou (ii) em abordagens de apoio ao desenvolvimento de tecnologia. $\mathrm{O}$ modelo TEACCH tem oito $(8 / 33)$ ocorrências, sendo uma associada ao método ABA [A32] e outra ao PECS [A29]. Destaca-se a adoção de TEACCH para soluções que visam alfabetização [A10 A12 A22 A24 A28] de pessoas com TEA, mostrando alinhamento destas pesquisas com as diretrizes do governo. Os métodos de comunicação alternativa CAA [A07 A16] e PECS [A01 A09 A19] foram menos numerosos, embora a maior parte das soluções (13/33) destine-se a trabalhar a competência de comunicação (Figura 4).

No Gráfico 1, "Outros" agrupa bases teóricas: da área de IHC [A06 A31], do desenvolvimento da criatividade [A02], das origens sociais de auto regulação [A05], da biologia da cognição e do paradigma da complexidade [A20], entre outros. $1 / 3$ dos artigos não explicita a base teórica que fundamenta a solução descrita. A ausência desta 
VII Congresso Brasileiro de Informática na Educação (CBIE 2018)

Anais do XXIX Simpósio Brasileiro de Informática na Educação (SBIE 2018)

informação dificulta conhecer quais as teorias que podem ser adotadas dentro do contexto de soluções tecnológicas para pessoas com autismo.

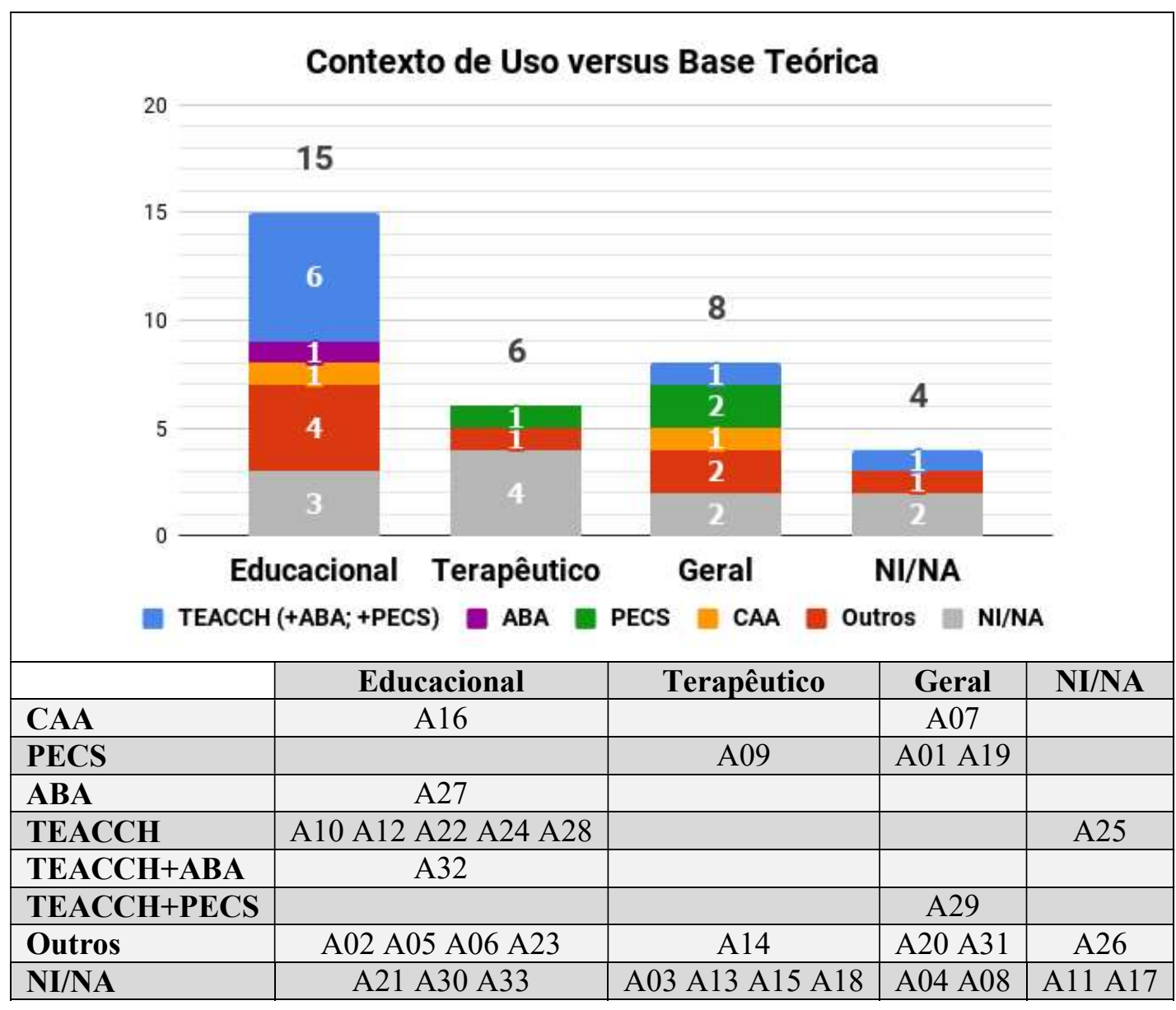

Gráfico 1. Distribuição quantitativa: Contexto de uso versus Base Teórica

\subsection{A Distribuição Geográfica das Pesquisas sobre TEA (QP06)}

Para a demonstração do quantitativo de publicações sobre TEA por estados brasileiros (Figura 5), foram consideradas as filiações dos autores dos artigos. As implantações dos Núcleos de Informática na Educação Especial na UFRGS e de Acessibilidade na UFAL podem ter contribuído para fomentar a pesquisa nos Estados.

Mesmo diante das recomendações de que a atuação junto às pessoas com TEA deve ser multidisciplinar [APA, 2013], a maioria das pesquisas foi realizada por apenas uma disciplina: Computação/Informática (17/33), Educação (2/33) e Engenharia Elétrica (1/33). Dentre as pesquisas que envolveram mais de uma disciplina se constatou a integração majoritária entre Computação e outras disciplinas (Educação/Pedagogia, Educação Física, Arquitetura, Engenharia, Matemática, Psicologia e Psiquiatria), assim como de Pedagogia e Enfermagem. A frequência da disciplina de Computação é esperada dado que uma das suas frentes de atuação é o desenvolvimento de soluções tecnológicas. 
VII Congresso Brasileiro de Informática na Educação (CBIE 2018)

Anais do XXIX Simpósio Brasileiro de Informática na Educação (SBIE 2018)

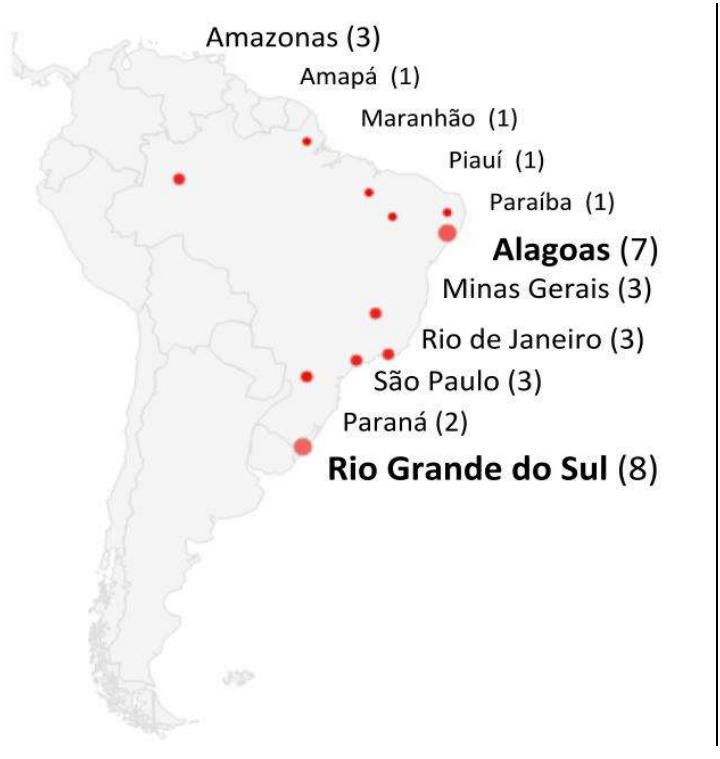

Rio Grande do Sul [A03 A04 A05 A07 A16 A20 A23 A33]; Alagoas [A10 A12 A13 A14 A15 A19 A24]; Amazonas [A06 A18 A25]; Minas Gerais [A01 A21 A29]; Rio de Janeiro [A08 A09 A11]; São Paulo [A02 A17 A26]; Paraná [A30 A32]; Amapá [A22]; Maranhão [A31]; Paraíba [A28]; Piauí [A27].

Figura 5. Distribuição Geográfica das Pesquisas sobre TEA por Estado Brasileiro

\section{Trabalhos Relacionados}

A revisão de literatura realizada por de Nunes et al. (2013) teve foco na inclusão educacional de pessoas com TEA no Brasil e contemplou 28 artigos (de 2008 a 2012) extraídos das bases de dados Scielo, Bireme e Capes. Como resultado os autores apontam a carência de estratégias pedagógicas específicas para a inclusão de pessoas com autismo e indicam que a inclusão pode ser potencializada com o desenvolvimento de recursos de Tecnologia Assistiva (TA). Os autores deixam uma lacuna por não mencionarem quais recursos de TA poderiam ser mediadores neste processo. Esta é reduzida com os resultados ora descritos, uma vez que, se fornece uma lista com soluções tecnológicas educacionais para pessoas com TEA. Granatto e colaboradores (2016) investigaram como as pessoas com deficiência podem se beneficiar do uso da tecnologia. A pesquisa considerou 91 artigos (de 1998 a 2015) oriundos dos anais do IHC e apenas um destes abordava TEA. Para a mesma conferência o atual estudo teve como retorno 8 artigos, sendo 4 deles incluídos na análise. Esta informação, assim como o quantitativo crescente de publicações sobre o tema (Figura 2), sugere maior interesse e dedicação dos pesquisadores brasileiros por esta área de conhecimento.

No contexto internacional a quantidade de revisões sobre o tema é mais abrangente. Grynszpan e colaboradores (2014) analisaram a eficácia pré e pós-uso de tecnologias por pessoas com autismo a partir de 21 publicações (de 1990 a 2011; PubMed, ERIC, IEEE, Web of Science). Os autores indicam evidências sobre a eficiência das intervenções com uso tecnologia. Este tipo de análise não é mencionada em nenhuma das 33 publicações contempladas nesta pesquisa. A realização de experimentos longitudinais para identificar o impacto do uso das tecnologias por pessoas com TEA se faz necessário para melhor direcionar o desenvolvimento de tais recursos. Fletcher-Watson (2014) foca no aprendizado assistido pelo computador por pessoas com autismo e analisa 52 artigos (1968 até 2011; PubMed). A autora pontua a importância do co-design entre projetistas de software e psicólogos, a relevância do design participativo e a pertinência de consultar diferentes especialistas. A revisão de Virnes e colaboradores (2015) inclui 255 artigos (de 2000 a 2010; ACM, EBSCO, ERIC, IEEE) para análise do uso de tecnologias por crianças com autismo. Os autores destacam a importância da multidisciplinaridade no 
VII Congresso Brasileiro de Informática na Educação (CBIE 2018)

Anais do XXIX Simpósio Brasileiro de Informática na Educação (SBIE 2018)

desenvolvimento recursos tecnológicos, de preferência com envolvimento ativo e longitudinal dos potenciais usuários da solução.

Considerando que no Brasil as políticas públicas destinadas à população com TEA e a difusão de informações sobre o tema são recentes, carecemos de estudos que permitam obter uma visão geral sobre "Autismo, Educação e Tecnologia" no âmbito nacional. Mapear essas informações permite que os interessados pelo tema conheçam os trabalhos desenvolvidos no Brasil, quem são os pesquisadores atuantes e quais são as disciplinas envolvidas, favorecendo a criação de uma rede de cooperação. Deste modo, oportunidades de desenvolvimento colaborativo e multidisciplinar se tornam mais viáveis, colaborando para o envolvimento de mais atores neste processo.

\section{Considerações Finais}

A adoção de recursos tecnológicos para auxiliar nas intervenções educacionais e terapêuticas junto às pessoas com TEA é uma realidade no contexto internacional. As vantagens da adoção da tecnologia se dá tanto pelo interesse dos indivíduos com TEA em seu uso, quanto na eficácia dos resultados obtidos. Além disso, é considerável a facilidade para personalizar as intervenções de forma adaptada para as necessidades de cada indivíduo. Considerando as recentes iniciativas do governo para assistir este público, é interessante mapear as pesquisas realizadas e difundidas no meio acadêmico nacional sobre "Autismo, Educação e Tecnologia". Informações sobre quais são as tecnologias disponíveis, em quais contextos podem ser utilizadas e para trabalhar quais competências, permitem conhecer como a comunidade acadêmica tem respondido à esta demanda da sociedade. Em adicional, favorece uma análise comparativa entre o panorama brasileiro face ao contexto internacional.

A revisão de literatura conduzida contemplou 33 artigos completos publicados em anais de eventos e revistas nacionais que veiculam pesquisas sobre Inclusão, Acessibilidade, Educação e Tecnologia. O resultado obtido permitiu identificar que as pesquisas realizadas estão em consonância com as diretrizes do governo, ou seja, há um expressivo conjunto de soluções de software destinados à Educação (principalmente para letramento e alfabetização) e que adotam os modelos práticos de intervenção consolidados nas abordagens clássicas (TEACCH, ABA, CAA e PECS). Entretanto, os sintomas de TEA são diversos e várias outras competências podem ser alvo das soluções tecnológicas a serem disponibilizadas para este público, a exemplo de: reconhecimento de expressões faciais emocionais e não emocionais, imitação, exploração visual, escrita manuscrita, coordenação motora, etc.

Embora a maioria das soluções seja direcionada para o público infantil, o que é coerente com a necessidade de diagnóstico e intervenções prematuras, os artigos não distinguem a idade cronológica da idade de desenvolvimento (real) das crianças, informação essencial na decisão sobre as práticas de intervenção. Outra informação relevante, e constantemente omitida nos artigos, diz respeito aos fundamentos teóricos que apoiam as pesquisas.

Por fim, face aos mapeamentos realizados sobre o tema em âmbito internacional, o quantitativo de publicações nacionais ainda é reduzido e estas não estão consolidadas enquanto pesquisas multidisciplinares e longitudinais. Para fortalecer esta área de pesquisa se faz necessário reunir esforços e promover a colaboração entre diferentes disciplinas, instituições e pesquisadores. Como trabalho futuro pretende-se mapear a rede 
VII Congresso Brasileiro de Informática na Educação (CBIE 2018)

Anais do XXIX Simpósio Brasileiro de Informática na Educação (SBIE 2018)

de colaboração entre pesquisadores e profissionais que se interessam pela tríade "Autismo, Educação e Tecnologia".

\section{Referências}

American Psychiatric Association. (2013). Diagnostic and statistical manual of mental disorders (DSM-5®). American Psychiatric Pub.

Brasil. Lei no 10.764, de 27 de dezembro de 2012. Disponível em: http://www.planalto.gov.br/ccivil_03/_ato2011-2014/2012/lei/112764.htm.

de Matos Cano, T. (2016), Panorama brasileiro do atendimento a autistas e necessidade da inclusão no censo 2020. Revista de Medicina e Saúde de Brasília, 5(2).

Fletcher-Watson, S. (2014), A targeted review of computer-assisted learning for people with autism spectrum disorder: Towards a consistent methodology. Review Journal of Autism and Developmental Disorders, 1(2), 87-100.

Gomes, V. N. e Machado, M. V. D. (2016). O acompanhamento terapêutico escolar no processo de inclusão de uma criança autista. Dissertação de Mestrado, Programa de Pós-Graduação em Psicologia, UFBA.

Granatto, F. C., Pallaro, M. A. e Bim, S. A. (2016). Digital Accessibility: Systematic Review of Papers from the Brazilian Symposium on Human Factors in Computer Systems. In Proceedings of the 15th IHC (p. 21). ACM.

Grynszpan, O., Weiss, P. L., Perez-Diaz, F. e Gal, E. (2014). Innovative technology-based interventions for autism spectrum disorders: a meta-analysis. Autism, 18(4), 346-361..

Mello, A. M., Andrade, M. A., Chen, H. H. e Dias, I. (2013) Retratos do autismo no Brasil. São Paulo: Associação dos Amigos do Autista.

Ministério da Saúde. (2013). Linha de cuidado para a atenção às pessoas com Transtornos do Espectro do Autismo e suas famílias na Rede de Atenção Psicossocial do SUS.

Nunes, D. R. P., Queiroz, O. A. e Schmidt, M. C. (2013). Inclusão educacional de pessoas com Autismo no Brasil: uma revisão da literatura. Revista Educação Especial, vol. 26, núm. 47, pp. 557-572

Rios, C., Ortega, F., Zorzanelli, R. e Nascimento L. F. (2015). From invisibility to epidemic: the narrative construction of autism in the Brazilian press. Interface (Botucatu); 19(53):325-35.

Rogé, B. (2010), Les interventions précoces pour les enfants avec autisme. Autisme et pratiques d'intervention, p. 23-58.

Vieira, N. M. e Baldin, S. R. (2017). Diagnóstico e Intervenção de Indivíduos com Transtorno do Espectro Autista. Encontro Internacional de Formação de Professores e Fórum Permanente de Inovação Educacional, 10(1).

Virnes, M., Kärnä, E. e Vellonen, V. (2015). Review of research on children with autism spectrum disorder and the use of technology. Journal of Special Education Technology, 30(1), 13-27. 